\title{
The use of probiotics in depression
}

\author{
Klaus W. Lange*, Yukiko Nakamura and Katharina M. Lange
}

Department of Experimental Psychology, University of Regensburg, Germany

*Corresponding author: Klaus W. Lange, Institute of Psychology, University of Regensburg, 93040 Regensburg, Germany. Tel: +49 941 9433815; Fax: +49 941 9434496; E-mail: klaus.lange@ur.de

DOI: $10.31665 /$ JFB.2020.12244

Received: December 14, 2020; Revised received \& accepted: December 26, 2020

Citation: Lange, K.W., Nakamura, Y., and Lange, K.M. (2020). The use of probiotics in depression. J. Food Bioact. 12: 3-8.

\begin{abstract}
Probiotics are thought to play a role in the extensive bidirectional communication between gut microbiota and the brain. A growing body of preclinical data suggests that probiotics may be effective in alleviating low mood and improving depressive symptoms. Preliminary evidence derived from studies in rodent models supports the view that single-strain or multi-strain preparations of Bifidobacterium and Lactobacillus are able to improve behaviors related to depression. The extent of antidepressant effects in humans with a clinical diagnosis of depression is less clear. While some support for the efficacy of probiotic supplementation has been found, the pooled effects in meta-analyses have generally been small. The positive effects reported for probiotics in major depression should be regarded as preliminary. Large, randomized trials assessing the efficacy of specific probiotic strain combinations over various time spans in individuals with clinically significant presentations of depression are needed to evaluate the therapeutic potential of probiotics. In light of accumulating evidence showing that probiotics may have beneficial effects in reducing depressive symptoms and may be useful as an adjunct intervention in major depressive disorder, the efficacy of probiotic foods in the prevention of depression should be investigated.
\end{abstract}

Keywords: Probiotics; Depression; Gut microbiome; Treatment; Prevention.

\section{Introduction}

Major depression is a common psychiatric disorder characterized by depressed mood or significantly reduced pleasure or interest in all activities (American Psychiatric Association, 2013). Major depressive disorder is highly debilitating, severely limiting psychosocial functioning and diminishing the quality of life of affected individuals (Malhi and Mann, 2018). The rate of recurrence is high and the treatment of depression poses a major challenge (Malhi and Mann, 2018). Up to $60 \%$ of individuals with major depressive disorder have been estimated to experience some degree of nonresponse to pharmacotherapy (Fava, 2003). Furthermore, major depression has been projected by the World Health Organization to rank as the first cause of global burden of disease by 2030 (World Health Organization, 2008). Subclinical levels of depressive symptoms can be observed in healthy populations (Gawlik et al., 2013).

Pharmacotherapy and psychotherapy are commonly used to treat people with depression. However, the ability of these treatment strategies to avert disease burden remains limited (e.g. Casacalenda et al., 2002). The need for the development of novel pharmacological compounds for depression has repeatedly been emphasized (e.g. Insel, 2015; Miller, 2010), and additional approaches in the prevention and treatment of depression are required. In view of this, nutritional psychiatry has developed as a new field of research, since diet quality and nutrition are important in the promotion of metabolic health (Bennett et al., 2015; Lange, 2017a; Lange, 2017b), and have also been shown to be involved in the regulation of mental health and to be modifiable risk factors for mental disorders (e.g. Lange, 2018a; Lange, 2020; Marx et al., 2017). For example, dietary patterns such as the Mediterranean diet, with limited amounts of processed foods and high consumption of fruit, vegetables, nuts, seeds, wholegrains and fish, have been found to be inversely correlated with the risk of depression (Lai et al., 2014; Opie et al., 2015; Psaltopoulou et al., 2013). In contrast, high-sugar and high-fat diets containing large amounts of processed foods (Western diets) appear to be positively associated with depression (Lai et al., 2014). A con- 
Table 1. Experimental findings supporting a role of the gut microbiome in the modulation of depressive symptoms

Development of depressive-like behaviors in germ-free mice inoculated with fecal samples of individuals with depression (Zheng et al., 2016)

Different composition of the intestinal microbiota of individuals with major depressive disorder compared to controls (Bravo et al., 2011; Jiang et al., 2015; Zheng et al., 2016)

Altered counts of both gram-positive and gram-negative bacteria in individuals with depression (Jiang et al., 2015; Naseribafrouei et al., 2014)

Link between alterations in the microbiome (e.g. Faecalibacterium) of individuals with depression and severity of depression (Jiang et al., 2015)

sistent association between diet, nutrition and depression in adults has been found in several systematic reviews and meta-analyses; demographic factors and reverse causality do not appear to explain this relationship (Lai et al., 2014; Li et al., 2017; Psaltopoulou et al., 2013). Several biological pathways implicated in mental disorders including depression can be influenced by diet. These pathways include oxidative stress, inflammation, mitochondrial dysfunction, neuronal plasticity, epigenetic changes and the intestinal microbiome (for review see Marx et al., 2017).

\section{Gut microbiota and brain function}

The microbiota is a community of commensal, symbiotic and pathogenic microorganisms, the majority of which live in the gastrointestinal tract (Gill et al., 2006). The human intestine is colonized by bacteria, archaea and eukaryotes, the number of which may approximate the number of cells in the human body (Sender et al., 2016). Genetic, epigenetic and especially dietary factors can influence the intestinal microbiota (Yadav et al., 2018). While symbiosis of the intestinal microbiota can contribute to the maintenance of undisturbed physiological conditions in the host, dysbiosis, i.e. an imbalance in gut microbiota, may be involved in the pathogenesis of various diseases, such as cardiovascular, gastrointestinal and immune-related diseases (Gourbeyre et al., 2011; Hungin et al., 2013; Khalesi et al. 2014) as well as mental disorders (e.g. Lange et al., 2020, Mohajeri et al., 2018).

Emerging evidence suggests a bidirectional interaction between the microbial communities in the gastrointestinal tract and the host central nervous system via the gut-brain axis (Cryan and Dinan, 2012; Dinan and Cryan, 2017a; Foster and Neufeld, 2013). Preclinical studies in animal models have found links between changes or deficits of the intestinal microbiota and neurochemical alterations in the brain, and the gut microbiota has been demonstrated to influence brain development, central functions and behaviors of the host (Bercik et al., 2011a; Crumeyrolle-Arias et al., 2014; Cryan and Dinan, 2012; Desbonnet et al., 2014; Diaz Heijtz et al., 2011; Dinan and Cryan, 2017b; Rogers et al., 2016). For example, an early study in germ-free mice observed a link between the gut microbiota and a reduction in brain-derived neurotrophic factor concentrations in the hippocampus and an elevated stress response in the hypothalamic-pituitary-adrenal axis (Sudo et al., 2004).

Other findings in mice, showing marked differences in the volume and dendritic morphology of the hippocampus and amygdala and between germ-free mice and conventionally colonized mice (Luczynski et al., 2016), suggest that the gut microbiome plays a key role in the development of normal brain morphology and ultrastructure of central neurons. The hypothesis that the gut microbiota can modulate brain function and modify behavior is supported by preclinical studies of germ-free animals, bacterial infections, fecal transplantation and probiotic treatment (Cryan and Dinan, 2012). Furthermore, intestinal microbial dysbiosis could be implicated in neuropsychiatric disorders, such as depression, bipolar disorder, schizophrenia and autism spectrum disorders (Lange et al., 2020; Rogers et al., 2016). For example, individuals with major depressive disorder appear to have distinct compositions of the gut microbiota in comparison with healthy people (Jiang et al., 2015; Naseribafrouei et al., 2014). Further findings in support of a role of the intestinal microbiota in the modulation of depressive symptoms in humans are presented in Table 1. These observations support the view that the intestinal microbiota could be a potential target for novel antidepressant interventions.

\section{Probiotics and depression}

\subsection{Animal studies}

Probiotics are live microorganisms whose consumption contributes to the intestinal microbial flora of the host, resulting in beneficial health effects (World Health Organization, 2001). In respect to the central nervous system, a number of behavioral experiments investigating potentially positive effects of probiotics on brain and behavior have been conducted in rodents. For example, colonizing germ-free mice with Bifidobacterium infantis has been shown to normalize their previously overreactive hypothalamic-pituitaryadrenal axis in response to restraint stress and to decrease their stress hormone levels to those found in control animals (Sudo et al., 2004). Various mechanisms have been suggested to be involved in the effects of probiotics on brain functions, including the regulation of mood and emotion (see Table 2).

Most of the available animal studies have used single-strain or multi-strain preparations of Bifidobacterium (B. breve, B. longum, B. infantis) and Lactobacillus (L. casei, L. helveticus, L. plantarum, L. rhamnosus) and explored their effects on rodents in tests assessing depression-like behaviors, such as the forced-swim, tail-suspension and sucrose-preference tests. Based on the findings in animal models, all of these preparations have been shown to improve behaviors related to depression (for review see Wang et al., 2016). However, the heterogeneity of the studies in regard to factors such as probiotic strains, dose and duration of probiotic administration, rodent strains, health conditions of animals and brain and behavioral functions assessed needs to be considered. Moreover, findings in preclinical rodent models of depression cannot be directly translated to clinical interventions in individuals with depression.

\subsection{Epidemiology}

A nationwide, large cross-sectional study conducted in Korea, in- 


\section{Table 2. Potential mechanisms involved in probiotic effects on the brain}

Increases in the diversity of intestinal microbiota and the composition of beneficial bacteria (Kwok et al., 2014; Smith et al., 2014; Wang et al., 2015)

Changes in food metabolites, such as short-chain fatty acids and tryptophan, due to "improvements" in intestinal microbiota (Desbonnet et al., 2008; Hsiao et al., 2013)

Effects on gut-brain interaction through the vagus and enteric nerves (Bercik et al., 2011b; Bravo et al., 2011)

Biochemical changes in the brain, including concentrations of brain-derived neurotrophic factor (O'Sullivan et al., 2011) and neurotransmitters, such as monoamines (Liu et al., 2016a, Liu et al., 2016b) and y-aminobutyric acid (Bravo et al., 2011)

Reduced corticosteroid levels due to attenuation of the stress response mediated by the hypothalamic-pituitary-adrenal axis (Ait-Belgnaoui et al., 2012)

Effects on the immune system by decreasing the production of pro-inflammatory cytokines and inflammation (Desbonnet et al., 2008; Desbonnet et al., 2010)

cluding 26,118 individuals aged 19-64 years, has recently investigated the association between depression status, as determined using a self-reported clinical diagnosis and a patient health questionnaire, and the consumption of probiotic foods, as assessed using a food frequency questionnaire (Kim and Shin, 2019). In comparison with the highest tertile of self-reported probiotic food intake, the lowest tertile had significantly higher odds in self-reported clinical depression and depression severity (Kim and Shin, 2019). These findings suggest that the consumption of probiotic foods might have beneficial effects on depression and could be capable of reducing the risk of depression in adults.

\subsection{Clinical trials}

In recent years, several systematic reviews and meta-analyses have analyzed the effects of probiotics on depression (see Table 3). Many of the randomized controlled trials conducted in this area have assessed the impact of probiotics on (subclinical) depressive symptoms in healthy people. The results of these trials, however, cannot be extrapolated to people with clinically diagnosed depression. Studies investigating supplementary probiotic interventions in the treatment of individuals with a clinical diagnosis of depression have recently been systematically reviewed (Noonan et al., 2020). All studies included in the review demonstrated significant improvements in one or more outcome measures of the impact of probiotics in comparison with placebo or no-treatment conditions or with assessments at baseline (Noonan et al., 2020). No negative effects or adverse events related to the use of probiotics were reported. However, this could reflect positive reporting bias, since systematic reporting of adverse events associated with probiotic interventions appears to be lacking (Gwee et al., 2018). Most of the studies were conducted in individuals with depression who were receiving antidepressant medication. Probiotics should therefore be considered as complementary to standard therapy rather than as an alternative. Taken together, the findings suggest that the administration of probiotics may be useful as an add-on treatment in clinical depression.

\section{Future directions}

In view of the evidence from the available findings of both animal and human studies, probiotics appear to be capable of improving brain function and depressive symptomatology (Wang et al., 2016). The results of preclinical experiments suggesting antidepressant efficacy of probiotics cannot be directly translated to clinical trials. In humans, various problems of the studies investigating the use of probiotics in depression need to be considered (see Table 4). Major limitations include small sample sizes, short durations of probiotic supplementation and the wide variety of single-strain and multiplestrain probiotics administered. The long-term efficacy of probiotics, in particular on the remission of depression, and lasting effects following discontinuation of their administration are unclear. Probiotic supplements appear to be well-tolerated, with low drop-out rates in the available studies (Nikolova et al., 2019). However, possible adverse events following extended use of probiotics are unknown.

In summary, our knowledge regarding the clinical effectiveness of probiotics in depression is insufficient. Therefore, further high-quality studies investigating their effects in major depressive disorder and determining optimal composition, dosage and duration of probiotic supplementation are warranted. It is noteworthy that in other fields of clinical medicine, such as the management of gastrointestinal disorders, the accumulation of evidence has often weakened rather than strengthened the case for the therapeutic use of probiotics (Preidis et al., 2020).

The mechanisms involved in the effects of probiotics on depression, in particular those related to stress, should be explored in more detail. A recent systematic review and meta-analysis, based on randomized controlled trials of the effects of probiotics on stress in healthy people, concluded that probiotics can decrease subjective stress levels in healthy individuals and could alleviate stressrelated subthreshold levels of depression and anxiety (Zhang et al., 2020). Most animal models used for the assessment of the effects of probiotics on behaviors related to depression cannot, for ethical reasons, be adapted to humans. However, future investigations may correlate gut microbiota composition with specific behaviors and manipulate the interaction between gut microbiota and central nervous system using probiotics.

The epidemiological findings from Korea, demonstrating a positive influence of the consumption of probiotic foods on depression (Kim and Shin, 2019), point to a preventive potential of probiotics. Such benefits would probably require an intake of probiotics over extended periods of time. A recommendation for consumption of probiotics may be made in view of the apparent lack of adverse events associated with their intake. However, scientific evaluation of this approach will be limited to observations, since a high-quality randomized study design is not feasible. Furthermore, self-treatment with dietary supplements without proven benefit is not recommended.

An important, though frequently neglected factor in the assessment of food bioactives in mental disorders is related to etiology and psychopathology. Biological, environmental, social and cultural factors are involved in the etiology of depression, and the diagnosis of depression relies on a number of symptoms, none of which are pathognomic of the condition (Malhi and Mann, 2018). 
Table 3. Systematic reviews and meta-analyses of the effects of probiotics on depression in humans

\begin{tabular}{ll}
\hline Authors & Type of study \\
\hline Huang et & Meta-analysis of randomized controlled trials
\end{tabular}

al., 2016

Pirbaglou et

al., 2016

Wallace and

Milev, 2017

$\mathrm{Ng}$ et al.,

2018

Goh et al., 2019

\begin{abstract}
Liu et al., Random-effects meta-analysis of 34 controlled
$2019 \quad$ clinical trials investigating the effects of prebiotics and probiotics on depression
\end{abstract}

Nikolova et Systematic review and meta-analysis of 3 randomized al., 2019 controlled trials of probiotics ( 2 studies of probiotics in addition to antidepressants and 1 study on probiotics as sole treatment) in clinical depression (229 participants)

Chao et Meta-analysis of randomized controlled trials al., 2020 evaluating the effectiveness of probiotics on depressive symptomatology in participants with a depressive disorder diagnosis

Noonan et Systematic review of probiotics as treatment al., 2020 for clinically diagnosed depression

\section{Conclusions}

Oral probiotic use showed a significant reduction in depression scale score in both individuals with major depressive disorder and healthy people. Beneficial effects were found in people aged 60 and below, but not in those aged 65 and older.

Limited support for positive impact of some probiotics on reducing depressive symptoms.

The majority of studies found positive effects on measures of depressive symptoms.

No significant effects of probiotic supplementation on mood compared to placebo. Subgroup analysis found significant mood improvements in individuals with mild-to-moderate depressive symptoms and non-significant effects in healthy people.

Treatment with probiotics significantly improved depressive symptoms compared to placebo. Significant beneficial effect of probiotics on depressive symptoms in people with major depressive disorder, but not in other clinical conditions and in the general population.

No significant difference between prebiotics and placebo; small but significant effects for probiotics, with larger effect in clinical/medical samples than community ones.

Possible beneficial effects of probiotics on depressive symptoms when used as supplement to antidepressants.

Significant alleviation of depressive symptoms in individuals with depression following administration of probiotics compared to placebo.

Significant improvements following probiotics in one or more outcome measures compared to placebo/ no treatment or to baseline measurements.
In short, depression is a clinically and probably pathophysiologically heterogeneous condition. Thus, as in other mental disorders, there is unlikely to be a one-fits-all solution in regard to the therapeutic efficacy of food bioactives such as probiotics (Lange, 2018b; Lange et al. 2020). The identification of subgroups of people who are most likely to benefit from probiotic supplementation may therefore be necessary.

\section{Conclusion}

Novel treatments are needed to reduce the global burden of ma-

Table 4. Limitations and problems of the available findings in respect to the use of probiotics in depression

Self-report of depressive symptoms versus clinically confirmed diagnosis of major depressive disorder.

Threshold of depression severity for inclusion (mild versus moderate).

Small sample sizes in studies of individuals with major depressive disorder.

Wide variety of composition of the single-strain and multiple-strain probiotics used.

Dose and duration of probiotic supplementation.

Use of probiotics as supplementation to pharmacological therapy or as sole treatment.

Degree of improved symptomatology: symptom rating scales versus remission of depression.

Different antidepressive effects of probiotics in different age groups.

Unclear long-term efficacy of probiotics during supplementation and following discontinuation. 
jor depression. The use of probiotics in depression has attracted increasing scientific and clinical interest in recent years. Both preclinical and clinical findings suggest that targeting the gut-microbiota-brain axis using probiotics may be a useful approach in reducing the severity of depression. The available evidence suggests that the role of intestinal microbiota and the use of probiotics in both the treatment and prevention of depression deserves more intensive investigation. However, the current evidence for probiotics in the therapy of major depression is modest. While some support for their efficacy has been found, the pooled effects in meta-analyses were generally small. Therefore, the positive effects observed for probiotics in major depression need to be regarded as preliminary. Large, randomized trials assessing the efficacy of specific probiotic strain combinations over various time spans in individuals with clinically significant presentations of depression are needed in order to evaluate the therapeutic potential of probiotics. In addition, the mechanisms underlying the probiotic effects on depression should be further elucidated.

\section{References}

Ait-Belgnaoui, A., Durand, H., Cartier, C., Chaumaz, G., Eutamene, H., Ferrier, L., Houdeau, E., Fioramonti, J., Bueno, L., and Theodorou, V. (2012). Prevention of gut leakiness by a probiotic treatment leads to attenuated HPA response to an acute psychological stress in rats. Psychoneuroendocrinology 37: 1885-1895.

American Psychiatric Association. (2013). Diagnostic and Statistical Manual of Mental Disorders, 5th ed. American Psychiatric Association, Washington, DC.

Bennett, B.J., Hall, K.D., Hu, F.B., McCartney, A.L., and Roberto, C. (2015). Nutrition and the science of disease prevention: a systems approach to support metabolic health. Ann. N. Y. Acad. Sci. 1352: 1-12.

Bercik, P., Denou, E., Collins, J., Jackson, W., Lu, J., Jury, J., Deng, Y., Blennerhassett, P., Macri, J., McCoy, K.D., Verdu, E.F., and Collins, S.M. (2011a). The intestinal microbiota affect central levels of brain-derived neurotropic factor and behavior in mice. Gastroenterology 141 : 599-609.

Bercik, P., Park, A.J., Sinclair, D., Khoshdel, A., Lu, J., Huang, X., Deng, Y., Blennerhassett, P.A., Fahnestock, M., Moine, D., Berger, B., Huizinga, J.D., Kunze, W., McLean, P.G., Bergonzelli, G.E., Collins, S.M., and Verdu, E.F. (2011b). The anxiolytic effect of Bifidobacterium longum NCC3001 involves vagal pathways for gut-brain communication. Neurogastroenterol. Motil. 23: 1132-1139.

Bravo, J.A., Forsythe, P., Chew, M.V., Escaravage, E., Savignac, H.M., Dinan, T.G., Bienenstock, J., and Cryan, J.F. (2011). Ingestion of Lactobacillus strain regulates emotional behavior and central GABA receptor expression in a mouse via the vagus nerve. Proc. Natl. Acad. Sci. U. S. A. 108: 16050-16055.

Casacalenda, N., Perry, J.C., and Looper, K. (2002). Remission in major depressive disorder: a comparison of pharmacotherapy, psychotherapy, and control conditions. Am. J. Psychiatry 159: 1354-1360.

Chao, L., Liu, C., Sutthawongwadee, S., Li, Y., Lv, W., Chen, W., Yu, L., Zhou, J., Guo, A., Li, Z., and Guo, S. (2020). Effects of probiotics on depressive or anxiety variables in healthy participants under stress conditions or with a depressive or anxiety diagnosis: A meta-analysis of randomized controlled trials. Front. Neurol. 11: 421.

Crumeyrolle-Arias, M., Jaglin, M., Bruneau, A., Vancassel, S., Cardona, A., Daugé, V., Laurent Naudon, L., and Rabot, S. (2014). Absence of the gut microbiota enhances anxiety-like behavior and neuroendocrine response to acute stress in rats. Psychoneuroendocrinology 42: 207-217.

Cryan, J.F., and Dinan, T.G. (2012). Mind-altering microorganisms: the impact of the gut microbiota on brain and behaviour. Nat. Rev. Neurosci. 13: 701.

Desbonnet, L., Clarke, G., Shanahan, F., Dinan, T.G., and Cryan, J.F. (2014). Microbiota is essential for social development in the mouse. Mol. Psychiatry 19: 146-148.

Desbonnet, L., Garrett, L., Clarke, G., Bienenstock, J., and Dinan, T.G.
(2008). The probiotic Bifidobacteria infantis: an assessment of potential antidepressant properties in the rat. J Psychiatr. Res. 43: 164-174.

Desbonnet, L., Garrett, L., Clarke, G., Kiely, B., Cryan, J.F., and Dinan, T.G. (2010). Effects of the probiotic Bifidobacterium infantis in the maternal separation model of depression. Neuroscience 170: 1179-1188.

Diaz Heijtz, R., Wang, S., Anuar, F., Qian, Y., Björkholm, B., Samuelsson, A. Hibberd, M.L., Forssberg, H., and Pettersson, S. (2011). Normal gut microbiota modulates brain development and behavior. Proc. Natl. Acad. Sci. 108: 3047-3052.

Dinan, T.G., and Cryan, J.F. (2017a). The microbiome-gut-brain-axis in health and disease. Gastroenterol. Clin. 46: 77-89.

Dinan, T.G., and Cryan, J.F. (2017b). Gut-brain axis in 2016: brain-gut-microbiota axis-mood, metabolism, and behaviour. Nat. Rev. Gastroenterol. Hepatol. 14: 69.

Fava, M. (2003). Diagnosis and definition of treatment-resistant depression. Biol. Psychiatry 53: 649-659.

Foster, J.A., and Neufeld, K.A.M. (2013). Gut-brain axis: how the microbiome influences anxiety and depression. Trends Neurosci. 36: 305312.

Gawlik, S., Waldeier, L., Muller, M., Szabo, A., Sohn, C., and Reck, C. (2013). Subclinical depressive symptoms during pregnancy and birth outcome-A pilot study in a healthy German sample. Arch. Womens Ment. Health 16: 93-100.

Gill, S.R., Pop, M., Deboy, R.T., Eckburg, P.B., Turnbaugh, P.J., Samuel, B.S., Gordon, J.I., Relman, D.A., Fraser-Liggett, C.M., and Nelson, K.E. (2006). Metagenomic analysis of the human distal gut microbiome. Science 312: 1355-1359.

Goh, K.K., Liu, Y.W., Kuo, P.H., Chung, Y.C.E., Lu, M.L., and Chen, C.H. (2019). Effect of probiotics on depressive symptoms: A meta-analysis of human studies. Psychiatry Res. 282: 112568.

Gourbeyre, P., Denery, S., and Bodinier, M. (2011). Probiotics, prebiotics, and synbiotics: impact on the gut immune system and allergic reactions. J. Leukoc. Biol. 89: 685-695.

Gwee, K.A., Lee, W.W.R., Ling, K.L., Ooi, C.J., Quak, S.H., Dan, Y.Y, Tien-Ho, K.T.H., Huang, J.G., Chua, A.S.B., Hilmi, I.N., Raja Ali, R.A., Ong, C., Simadibrata, M., Abdullah, M., Sollano, J.D., Leelakusolvong, S., Gonlachanvit, S., Lee, Y.Y., Ricaforte-Campos, J.D., Yin, Y.K., Chong, K.M., and Wong, C.Y. (2018). Consensus and contentious statements on the use of probiotics in clinical practice: a South East Asian gastro-neuro motility association working team report. J. Gastroenterol. Hepatol. 33: 1707-1716.

Hsiao, E.Y., McBride, S.W., Hsien, S., Sharon, G., Hyde, E.R., McCue, T., Codelli, J.A., Chow, J., Reisman, S.E., Petrosino, J.F., Patterson, P.H., and Mazmania, S.K. (2013). Microbiota modulate behavioral and physiological abnormalities associated with neurodevelopmental disorders. Cell 155: 1451-1463.

Huang, R., Wang, K., and Hu, J. (2016). Effect of probiotics on depression: A systematic review and meta-analysis of randomized controlled trials. Nutrients 8: 483.

Hungin, A.P., Mulligan, C., Pot, B., Whorwell, P., Agréus, L., Fracasso, P., Lionis, C., Mendive, J., Philippart de Foy, J.-M., Rubin, G., Winchester, C., and de Wit, N. (2013). Systematic review: probiotics in the management of lower gastrointestinal symptoms in clinical practice - an evidence-based international guide. Aliment. Pharmacol. Ther. 38: 864-886.

Insel, T.R. (2015). The NIMH experimental medicine initiative. World Psychiatry 14: 151-153.

Jacka, F.N., Pasco, J.A., Mykletun, A., Williams, L.J., Hodge, A.M., O’Reilly, S.L., Nicholson, G.C., Kotowicz, M.A., and Berk, M. (2010). Association of Western and traditional diets with depression and anxiety in women. Am. J. Psychiatry 167: 305-311.

Jiang, H., Ling, Z., Zhang, Y., Mao, H., Ma, Z., Yin, Y., Wang, W., Tang, W., Tan, Z., Shi, J., Li, L., and Ruan, B. (2015). Altered fecal microbioata composition in patients with major depressive disorder. Brain Behav. Immun. 48: 186-194.

Khalesi, S., Sun, J., Buys, N., and Jayasinghe, R. (2014). Effect of probiotics on blood pressure: a systematic review and meta-analysis of randomized controlled trials. Hypertension 64: 897-903.

Kim, M.S., and Shin, D.M. (2019). Probiotic food consumption is associated with lower severity and prevalence of depression: A nationwide cross-sectional study. Nutrition 63-64: 169-174.

Kwok, L.Y., Wang, L., Zhang, J., Guo, Z., and Zhang, H. (2014). A pilot study 
on the effect of Lactobacillus casei Zhang on intestinal microbiota parameters in Chinese subjects of different age. Benef. Microbes 5: 295-304.

Lai, J.S., Hiles, S., Bisquera, A., Hure, A.J., McEvoy, M., and Attia, J. (2014). A systematic review and meta-analysis of dietary patterns and depression in community-dwelling adults. Am. J. Clin. Nutr. 99: 181-197.

Lange, K.W. (2017a). The International Movement and Nutrition Society and the prevention of disease. Mov. Nutr. Health Dis. 1: 0.

Lange, K.W. (2017b). Movement and nutrition in health and disease. Mov. Nutr. Health Dis. 1: 1-2.

Lange, K.W. (2018a). Diet, exercise, and mental disorders-public health challenges of the future. Mov. Nutr. Health Dis. 2: 39-59.

Lange, K.W. (2018b). Do food bioactives play a role in attention-deficit/ hyperactivity disorder? J. Food Bioact. 4: 1-7.

Lange, K.W. (2020). Omega-3 fatty acids and mental health. Glob. Health J. $4: 18-30$.

Lange, K.W., Lange, K.M., Nakamura, Y., and Kanaya, S. (2020). Is there a role of gut microbiota in mental health? J. Food Bioact. 9: 4-9.

Li, S., Zhao, Y., Zhang, Y., Zhang, X., Huang, L., Li, D., Niu, C., ZhYang, Z., and Wang, Q. (2012). Antioxidant activity of Lactobacillus plantarum strains isolated from traditional Chinese fermented foods. Food Chem. 135: 1914-1919.

Li, Y., Lv, M.R., Wie, Y.J., Sun, L., Zhang, J.X., Zhang, H.G., and Li, B. (2017). Dietary patterns and depression risk: a meta-analysis. Psychiatry Res. 253: 373-382.

Liu, R.T., Walsh, R.F.L., and Sheeha, A.E. (2019). Prebiotics and probiotics for depression and anxiety: A systematic review and meta-analysis of controlled clinical trials. Neurosci. Biobehav. Rev. 102: 13-23.

Liu, W.H., Chuang, H.L., Huang, Y.T., Wu, C.C., Chou, G.T., Wang, S., and Tsai, Y.C. (2016a). Alteration of behavior and monoamine levels attributable to Lactobacillus plantarum PS128 in germ-free mice. Behav. Brain Res. 298: 202-209.

Liu, Y.W., Liu, W.H., Wu, C.C., Juan, Y.C., Wu, Y.C., Tsai, H.P., Wang, S., and Tsai, Y.C. (2016b). Psychotropic effects of Lactobacillus plantarum PS128 in early life-stressed and naïve adult mice. Brain Res. 1631: 1-12.

Luczynski, P., Whelan, S.O., O’Sullivan, C., Clarke, G., Shanahan, F., Dinan, T.G., and Cryan, J.F. (2016). Adult microbiota-deficient mice have distinct dendritic morphological changes: differential effects in the amygdala and hippocampus. Eur. J. Neurosci. 44: 2654-2666.

Malhi, G.S., and Mann, J.J. (2018). Depression. Lancet 392: 2299-2312.

Marx, W., Moseley, G., Berk, M., and Jacka, F. (2017). Nutritional psychiatry: the present state of the evidence. Proc. Nutr. Soc. 76: 427-436.

Miller, G. (2010). Is pharma running out of brainy ideas? Science 329: 502-504.

Mohajeri, M.H., La Fata, G., Steinert, R.E., and Weber, P. (2018). Relationship between the gut microbiome and brain function. Nutr. Rev. 76: 481-496.

Naseribafrouei, A., Hestad, K., Avershina, E., Sekelja, M., Linløkken, A., Wilson, R., and Rudi, K. (2014). Correlation between the human fecal microbiota and depression. Neurogastroenterol. Motil. 26: 1155-1162.

Ng, Q.X., Peters, C., Ho, C.Y.X., Lim, D.Y., and Yeo, W.S. (2018). A metaanalysis of the use of probiotics to alleviate depressive symptoms. J. Affect. Disord. 228: 13-19.

Nikolova, V., Zaidi, S.Y., Young, A.H., Cleare, A.J., and Stone, J.M. (2019). Gut feeling: randomized controlled trials of probiotics for the treatment of clinical depression: Systematic review and meta-analysis. Ther. Adv. Psychopharmacol. 9: 1-7.

Noonan, S., Zaveri, M., Macaninch, E., and Marty, K. (2020). Food \& mood: A review of supplementary prebiotic and probiotic interventions in the treatment of anxiety and depression in adults. BMJ Nutr. Prev. Health. doi:10.1136/bmjnph-2019-000053.

O'Sullivan, E., Barrett, E., Grenham, S., Fitzgerald, P., Stanton, C., Ross, R.P., Quigley, E.M.M., Cryan, J.F., and Dinan, T.G. (2011). BDNF expression in the hippocampus of maternally separated rats: does Bifidobacterium breve 6330 alter BDNF levels? Benef. Microbes 2: 199-207.

Opie, R.S., O'Neil, A., Itsiopoulos, C., and Jacka, F.N. (2015). The impact of whole-of-diet interventions on depression and anxiety: a systematic review of randomised controlled trials. Public Health Nutr. 18: 2074-2093.

Pirbaglou, M., Katz, J., de Souza, R.J., Stearns, J.C., Motamed, M., and Ritvo, P. (2016). Probiotic supplementation can positively affect anxiety and depressive symptoms: a systematic review of randomized controlled trials. Nutr. Res. 36: 889-898.

Preidis, G.A., Weizman, A.V., Kashyap, P.C., and Morgan, R.L. (2020). AGA technical review on the role of probiotics in the management of gastrointestinal disorders. Gastroenterology 159: 708-738.

Psaltopoulou, T., Sergentanis, T.N., Panagiotakos, D.B., Sergentanis, I.N., Kosti, R., and Scarmeas, N. (2013). Mediterranean diet, stroke, cognitive impairment, and depression: a meta-analysis. Ann. Neurol. 74: 580-591.

Rogers, G., Keating, D., Young, R., Wong, M., Licinio, J., and Wesselingh, S. (2016). From gut dysbiosis to altered brain function and mental illness: mechanisms and pathways. Mol. Psychiatry 21: 738.

Sánchez-Villegas, A., Delgado-Rodríguez, M., Alonso, A., Schlatter, J., Lahortiga, F., Majem, L.S., and Martínez-González, M.A. (2009). Association of the Mediterranean dietary pattern with the incidence of depression: the Seguimento Universidad de Navarra/University of Navarra follow-up (SUN) cohort. Arch. Gen. Psychiatry 66: 1090-1098.

Sender, R., Fuchs, S., and Milo, R. (2016). Revised estimates for the number of human and bacteria cells in the body. PLoS Biol. 14: e1002533.

Smith, C.J., Emge, J.R., Berzins, K., Lung, L., Khamishon, R., Shah, P., Rodrigues, D.M., Sousa, A.J., Reardon, C., Sherman, P.M., Barrett, K.E., and Gareau, M.G. (2014). Probiotics normalize the gut-brain-microbiota axis in immunodeficient mice. Am. J. Physiol. Gastrointest. Liver Physiol. 307: G793-G802.

Sudo, N., Chida, Y., Aiba, Y., Sonoda, J., Oyama, N., Yu, X.N., Kubo, C., and Koga, Y. (2004). Postnatal microbial colonization programs the hypothalamic-pituitary-adrenal system for stress response in mice. J. Physiol. 558: 263-275.

Wallace, C.J.K., and Milev, R. (2017). The effects of probiotics on depressive symptoms in humans: a systematic review. Ann. Gen. Psychiatry 16: $1-10$.

Wang, H., Lee, I.S., Braun, C., and Enck, P. (2016). Effect of probiotics on central nervous system functions in animals and humans: A systematic review. J. Neurogastroenterol. Motil. 22: 589-605.

Wang, T., Hu, X., Liang, S., Li, W., Wu, X., Wang, L., and Jin, F. (2015). Lactobacillus fermentum NS9 restores the antibiotic induced physiological and psychological abnormalities in rats. Benef. Microbes 6: 707-717.

World Health Organization. (2001). Evaluation of health and nutritional properties of powder milk and live lactic acid bacteria. Food and Agriculture Organization of the United Nations and World Health Organization Expert Consultation Report. pp. 1-34.

World Health Organization. (2008). The global burden of disease: 2004 update World Health Organization, Geneva.

Yadav, M., Verma, M.K., and Chauhan, N.S. (2018). A review of metabolic potential of human gut microbiome in human nutrition. Arch. Microbiol. 200: 203-217.

Zhang, N., Zhang, Y., Li, M., Wang, W., Liu, Z., Xi, C., Huang, X., Liu, J., Huang, J., Tian, D., Mu, J., Liao, X., and Zhai, S. (2020). Efficacy of probiotics on stress in healthy volunteers: A systematic review and meta-analysis based on randomized controlled trials. Brain Behav. 10: e01699.

Zheng, P., Zeng, B., Zhou, C., Liu, M., Fang, Z., Xu, X., Zeng, L., Chen, J., Fan, S., Du, X., Zhang, X., Yang, D., Yang, Y., Meng, H., Li, W., Melgiri, N.D., Licinio, J., Wei, H., and Xie, P. (2016). Gut microbiome remodeling induces depressive-like behaviors through a pathway mediated by the host's metabolism. Mol. Psychiatry 21: 786-796. 